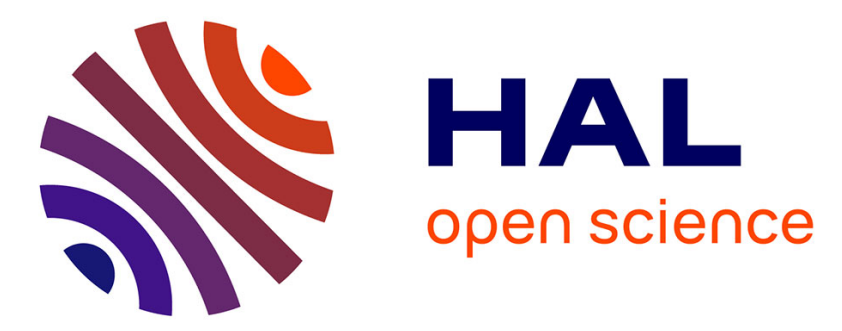

\title{
The Semi-Lagrangian Method for the Numerical Resolution of the Vlasov Equation
}

Eric Sonnendrucker, Jean Roche, Pierre Raphaël Bertrand, Alain Ghizzo

\section{To cite this version:}

Eric Sonnendrucker, Jean Roche, Pierre Raphä̈l Bertrand, Alain Ghizzo. The Semi-Lagrangian Method for the Numerical Resolution of the Vlasov Equation. Journal of Computational Physics, 1999, 149 (2), pp.201-220. 10.1006/jcph.1998.6148 . hal-01791851

\section{HAL Id: hal-01791851 \\ https://hal.univ-lorraine.fr/hal-01791851}

Submitted on 20 May 2018

HAL is a multi-disciplinary open access archive for the deposit and dissemination of scientific research documents, whether they are published or not. The documents may come from teaching and research institutions in France or abroad, or from public or private research centers.
L'archive ouverte pluridisciplinaire HAL, est destinée au dépôt et à la diffusion de documents scientifiques de niveau recherche, publiés ou non, émanant des établissements d'enseignement et de recherche français ou étrangers, des laboratoires publics ou privés. 


\title{
RINRIA
}

\section{The Semi-Lagrangian Method for the Numerical Resolution of Vlasov Equations}

\author{
Eric Sonnendrücker, Jean Roche, Pierre Bertrand, Alain Ghizzo \\ Thème 4 - Simulation et optimisation \\ de systèmes complexes \\ Projet Numath
}

Rapport de recherche $n^{\circ} 3393$ - Mars 1998 - 23 pages

\begin{abstract}
The numerical resolution of kinetic equations and in particular of Vlasov type equations is most of the time performed using PIC (Particle In Cell) methods which consist in describing the time evolution of the equation through a finite number of particles which follow the characteristic curves of the equation, the interaction with the external and self consistent fields being resolved using a grid. Another approach consists in computing directly the distribution function on a grid by following the characteristics backward in time for one time step and interpolating the value at the feet of the characteristics using the grid points values of the distribution function at the previous time step. In this report we introduce this last method and its use for different types of Vlasov equations.
\end{abstract}

Key-words: Vlasov equations, kinetic equations, semi-Lagrangian method, time splitting.

Unité de recherche INRIA Lorraine Technopôle de Nancy-Brabois, Campus scientifi que, 615 rue de Jardin Botanique, BP 101, 54600 VILLERS LÈS NANCY (France)

Téléphone : (33) 0383593030 - Télécopie : (33) 0383278319 Antenne de Metz, technopôle de Metz 2000, 4 rue Marconi, 55070 METZ

Téléphone : (33) 0387203500 - Télécopie : (33) 0387763977 


\section{La méthode semi-Lagrangienne pour la résolution numérique des équations de Vlasov}

Résumé : La r'esolution numerique des 'equations cin'etique comme les 'equations de Vlasov est la plupart du temps r'ealis'ee de nos jours par des m'ethodes PIC (Particle In Cell) qui consistent à d'ecrire 1 ' 'evolution en temps de 1 ''equation à travers un nombre fini de particules, les trajectoires des particules suivant les courbes caract'eristiques de l' equation de Vlasov, l'interaction avec les champs ext'erieurs et auto-consistant se faisant en utilisant un maillage. Une autre approche consiste à calculer directement la fonction de distribution solution de 1'equation de Vlasov sur un maillage en remontant les courbes caract 'eristiques sur un pas de temps à partir des points du maillage et en interpolant la fonction de distribution ant'erieure aux pieds de ces caract'eristiques. Dans ce rapport nous pr'esentons cette deuxième m'ethode et son utilisation pour diff'erents types d'equations de Vlasov.

Mots-clé : Equations de Vlasov, 'equations cinetiques, methode semi-Lagrangienne, time splitting. 


\section{Introduction}

The numerical resolution of kinetic equations the solution of which depends in addition to the time on three space variables and on three velocity variables is performed most of the time using ParticleIn-Cell (PIC) methods, which enable to get satisfying results with relatively few particles. However, for some applications, in particular when particles in the tail of the distribution play an important physical role or when the numerical noise due to the finite number of super-particles becomes too important, methods which compute the solution on a grid in phase space have proven to better describe the physics [1], [2], [3]. Such methods are all the more interesting when using parallel computers as they are, unlike PIC methods, very scalable [4].

In order to compute the solution of the one dimensional Vlasov equation

$$
\frac{\partial f}{\partial t}+v \frac{\partial f}{\partial x}+E(x, t) \frac{\partial f}{\partial v}=0
$$

coupled with Poisson's equation

$$
\frac{\partial E}{\partial x}=\int_{-\infty}^{+\infty} f(x, v, t) d v-1
$$

for $E$, the following procedure, originally introduced by Cheng and Knorr [5] and making full use of time-splitting, can be used to go from time step $t_{n}$ to $t_{n+1}$

1. Perform a half time step shift along the $x$-axis $f^{*}(x, v)=f^{n}(x-v \Delta t / 2, v)$

2. Compute the electric field at time $t_{n+1 / 2}$ by substituting $f^{*}$ in the Poisson equation (2)

3. Perform a shift along the $v$-axis $f^{* *}(x, v)=f^{*}\left(x, v-E\left(x, t_{n+1 / 2}\right) \Delta t\right)$

4. Perform a second half time step shift along the $x$-axis $f^{n+1}(x, v)=f^{* *}(x-v \Delta t / 2, v)$.

This method, called Eulerian because it used a phase space grid instead of particles, proved to work very well in this case. Moreover, the shifts along $x$ or $v$ are nothing but Lagrangian advections since the method is equivalent to solving the characteristics of the Vlasov equation. The advantage is that the scheme can use larger time steps than explicit Eulerian ones, the price to pay is to reconstruct a regular grid using interpolation. However, it could not be applied as easily to other kind of Vlasov problems like for instance the guiding-centre approximation

$$
\frac{\partial f}{\partial t}+\frac{\mathbf{E}(\mathbf{x}, t) \times \mathbf{B}}{B^{2}} \cdot \nabla_{x} f=0
$$

coupled with Poisson's equation, where the advection term $\mathbf{E}(\mathbf{x}, t) \times \mathbf{B} / B^{2}$ depends on $\mathbf{x}$ and the time-splitting method can not be applied, or a reduced relativistic Vlasov equation like

$$
\frac{\partial f}{\partial t}+\frac{p}{\gamma} \frac{\partial f}{\partial x}+\left(E_{x}-\frac{1}{2 \gamma} \frac{\partial}{\partial x}\left(A_{\perp}^{2}(x, t)\right)\right) \frac{\partial f}{\partial p}=0
$$

where

$$
\gamma=\sqrt{1+p^{2}+A_{\perp}^{2}(x, t)}
$$

coupled with Maxwell's equations for the vector potential $A_{\perp}$, where a similar problem occurs. These problems pushed us to try and extend the scope of application of the method. This could be RR $n^{\circ} 3393$ 
done, using the so-called semi-Lagrangian method or backward characteristics method which had already been investigated by the fluid dynamics community and especially in climate simulation [6]. A mathematical analysis of the method has also been performed by Bermejo [7].

This paper is organised as follows. In the next section we are going to present a number of Vlasov equations that we would like to be able to solve with our method, this will lead to an abstract formulation which will enclose all those equations. Then we shall introduce the semi-Lagrangian method for our abstract formulation and discuss some simplifications in special cases. After that we shall describe the implementation of the method in the two-dimensional case and finally numerical results will be presented.

\section{Different types of Vlasov equations}

The ultimate model we would like to use in order to describe the behaviour of a plasma is the relativistic three-dimensional Vlasov model which reads, for instance for an electron gas with $e=$ $m=1$,

$$
\frac{\partial f}{\partial t}+\frac{\mathbf{p}}{\gamma} \cdot \nabla_{x} f+\left(\mathbf{E}+\frac{\mathbf{p}}{\gamma} \times \mathbf{B}\right) \cdot \nabla_{p} f=0
$$

$\gamma$ being the Lorentz factor $\left(1+p_{x}^{2}+p_{y}^{2}+p_{z}^{2}\right)^{1 / 2}$, or its non-relativistic counterpart

$$
\frac{\partial f}{\partial t}+\mathbf{v} \cdot \nabla_{x} f+(\mathbf{E}+\mathbf{v} \times \mathbf{B}) \cdot \nabla_{v} f=0
$$

coupled to Maxwell's equations or some approximation of those. However, this would require a a six-dimensional grid, and as a minimum of points are required in each direction to represent the physics correctly, this would be too large even for the largest multiprocessor computer available today. Therefore, we shall investigate reduced models, which are adequate for describing specific physical problems.

The simplest model we are interested in, beyond the 1D electrostatic model, is the $2 \mathrm{D}$ electrostatic one which reads

$$
\frac{\partial f}{\partial t}+\mathbf{v} \cdot \nabla_{x} f+\mathbf{E}(\mathbf{x}, t) \cdot \nabla_{v} f=0
$$

coupled with Poisson's equation

$$
-\Delta \phi=1-\int f d \mathbf{v}
$$

where $\mathbf{E}=-\nabla \phi$.

The next category of models is motivated by the important problems of the non linear interaction of high intensity ultra short laser pulses with plasmas, with specific application to particle acceleration or inertial confinement fusion purpose. It consists of $1 \frac{1}{2} D, 1 \frac{2}{2} D$ and $2 \frac{1}{2} D$ electromagnetic models

$$
\frac{\partial f}{\partial t}+\frac{\mathbf{p}}{\gamma} \cdot \nabla_{x} f+\left(\mathbf{E}+\frac{\mathbf{p}}{\gamma} \times \mathbf{B}\right) \cdot \nabla_{p} f=0
$$

where compared to the full relativistic model (5) $f, \mathbf{E}$ and $\mathbf{B}$ depend only on one or two space variables and $f$ depends on two or three momentum components. Moreover, some components of the fields may vanish according to the wave polarisation, and the remaining are computed using some reduced set of Maxwell's equations. 
For the case of a laser wave propagating in one direction (say $x$ ), $f$ depends only on $x, p_{x}$ and $\mathbf{p}_{\perp}$. So one can further reduce the relativistic Vlasov (5) equation by looking for a specific class of exact solutions of the form, say in $1 \frac{2}{2} D$,

$$
f\left(x, p_{x}, p_{y}, p_{z}, t\right)=F\left(x, p_{x}, t\right) \delta\left(p_{y}-P_{y}(x, t)\right) \delta\left(p_{z}-P_{z}(x, t)\right) .
$$

Then $F$ is solution of

$$
\frac{\partial F}{\partial t}+\frac{p_{x}}{\gamma} \frac{\partial F}{\partial x}+\left(E_{x}+\frac{P_{y} B_{z}-P_{z} B_{y}}{\gamma}\right) \frac{\partial F}{\partial p_{x}}=0,
$$

with $\gamma=\left(1+p_{x}^{2}+P_{y}(x, t)^{2}+P_{z}(x, t)^{2}\right)^{1 / 2}$. This results from the conservation of the generalised canonical momentum along the wave plane, i.e. $P_{y}-A_{y}=$ const and $P_{z}-A_{z}=$ const. Deriving with respect to time and using

we get

$$
\mathbf{E}_{\perp}=-\frac{\partial \mathbf{A}_{\perp}}{\partial t}
$$

$$
\frac{\partial P_{y}}{\partial t}=E_{y}, \quad \frac{\partial P_{z}}{\partial t}=E_{z} .
$$

This mono-kinetic perpendicular description in the longitudinal axis is consistent with the very low temperature of the targets ( $\mathrm{a}$ few $\mathrm{eV}$ ) while the longitudinal electron temperature can reach the $\mathrm{MeV}$ range during the interaction. Finally, using $\mathbf{B}_{\perp}=\operatorname{curl} \mathbf{A}_{\perp}$, we find that

$$
P_{y} B_{z}-P_{z} B_{y}=\frac{1}{2} \frac{\partial A_{\perp}^{2}}{\partial x}
$$

and we recover Eq. (4).

Instead of reducing the dimension or assuming a specific form for the solution one can also obtain another type of Vlasov equation by averaging over the magnetic orbits in a highly magnetised plasma like a tokamak plasma, this yields the drift-kinetic model which reads, in the case of a uniform external magnetic field,

$$
\frac{\partial f}{\partial t}+\left(\mathbf{v}_{\|}+\frac{\mathbf{E} \times \mathbf{B}}{B^{2}}\right) \cdot \nabla_{x} f+\mathbf{E}_{\|} \cdot \nabla_{v} f=0 .
$$

If the computational box is reduced to the plane perpendicular to $\mathbf{B}$, the drift-kinetic equation reduces to the guiding-centre equation (3).

Finally, some simplification of the Vlasov equation may be obtained in some cases by using some other coordinate system. One example of this case is the $1 \frac{1}{2} D$ axisymmetric model where $f=f\left(r, v_{r}, v_{\theta}, t\right)$ for which we have

$$
\frac{\partial f}{\partial t}+v_{r} \frac{\partial f}{\partial r}+\left(E_{r}+\frac{v_{\theta}^{2}}{r}\right) \frac{\partial f}{\partial v_{r}}-\frac{v_{r} v_{\theta}}{r} \frac{\partial f}{\partial v_{\theta}}=0 .
$$

All the types of Vlasov equations we have listed above can be written in the following way

$$
\frac{\partial f}{\partial t}+U(X, t) \cdot \nabla_{X} f=0
$$

where $X$ stands for the phase space coordinates and $U$ is a divergence free vector field having up to six components in the full three-dimensional case. For example, in the case of the 3D non relativistic Vlasov equation, $X=\left(x, y, z, v_{x}, v_{y}, v_{z}\right)$ and $U(X, t)=\left(v_{x}, v_{y}, v_{z}, E_{x}+v_{y} B_{z}-\right.$ $\left.v_{z} B_{y}, E_{y}+v_{z} B_{x}-v_{x} B_{z}, E_{z}+v_{x} B_{y}-v_{y} B_{x}\right)$, all components of the electric and magnetic field depending on $x, y, z$ and $t$.

$\mathrm{RR} \mathrm{n}^{\circ} 3393$ 


\section{The time-splitting problem}

For an advection field $U$ which is divergence free, as it happens for our Vlasov equations, equation (9) can also be written in conservative form

$$
\frac{\partial f}{\partial t}+\operatorname{div}_{X}(U(X, t) f)=0
$$

For numerical purposes, conservation laws like (10), can be further reduced by splitting. Indeed, splitting the components of $X$ in two sets $X_{1}$ and $X_{2},(10)$ can be written

$$
\frac{\partial f}{\partial t}+\operatorname{div}_{X_{1}}\left(U_{1}\left(X_{1}, X_{2}, t\right) f\right)+\operatorname{div}_{X_{2}}\left(U_{2}\left(X_{1}, X_{2}, t\right) f\right)=0 .
$$

Moreover, it has been verified, see for example [8], that one can use a second order in time numerical scheme for solving separately at each time step

$$
\frac{\partial f}{\partial t}+\operatorname{div}_{X_{1}}\left(U_{1}\left(X_{1}, X_{2}, t\right) f\right)=0
$$

and

$$
\frac{\partial f}{\partial t}+d i v_{X_{2}}\left(U_{2}\left(X_{1}, X_{2}, t\right) f\right)=0
$$

and keep the second order accuracy for the whole equation (11) by alternating the solves.

For our purposes, this will be useful in the case where both $\operatorname{div}_{X_{1}} U_{1}\left(X_{1}, X_{2}, t\right)=0$ and div $v_{X_{2}} U_{2}\left(X_{1}, X_{2}, t\right)=0$, as in this case $U_{1}$ and $U_{2}$ can be taken out of the div operator and yield the traditional advective form.

Applying this, first for the non-relativistic Vlasov equation (6), we notice that $\partial_{i} v_{i}=0$ and $\partial_{v_{i}}(\mathbf{E}+\mathbf{v} \times \mathbf{B})_{i}=0, i$ standing for $x, y$ or $z$. Hence, it is possible to split (6) into six 1D advections. This method has already been applied in the $1 \mathrm{D}$ case, as we mentioned in the introduction and also in the 2D case [9], [10].

In the case of the relativistic Vlasov equation (5), we still have obviously $\partial_{i} p_{i} / \gamma=0$, but no such property for the force term which now depends on the Lorentz factor $\gamma=\sqrt{1+p_{x}^{2}+p_{y}^{2}+p_{z}^{2}}$.

However, a straightforward computation shows that we still have $d i v_{p}\left(\mathbf{E}+\frac{\mathbf{p}}{\gamma} \times \mathbf{B}\right)=0$. Hence we could split the equation into one 3D advection for the momentum space and three $1 \mathrm{D}$ advection for the physical space. Whether the splitting of the physical space could be a problem needs some further investigations, as it results in a loss of isotropy having three special directions. Finally, in the other models we introduced, splitting does not seem justified a priori and a full multidimensional advection scheme for Eq. (9) is needed.

\section{The semi-Lagrangian method}

Let us now introduce the semi Lagrangian method on the abstract model (9). For this, we need to introduce the characteristics of (9), which are the solutions of the dynamical system

$$
\frac{d \mathbf{X}}{d t}=\mathbf{U}(\mathbf{X}(t), t)
$$


Let us denote by $\mathbf{X}(t ; \mathbf{x}, s)$ the solution at time $t$ whose value is $\mathbf{x}$ at time $s$. Taking $\mathbf{X}(t)$ a solution of (12), we have

$$
\begin{aligned}
\frac{d}{d t}(f(\mathbf{X}(t), t)) & =\frac{\partial f}{\partial t}+\frac{d \mathbf{X}}{d t} \cdot \nabla_{X} f \\
& =\frac{\partial f}{\partial t}+\mathbf{U}(\mathbf{X}(t), t) \cdot \nabla_{X} f=0
\end{aligned}
$$

which means that $f$ is constant along the characteristics. This can also be written

$$
f(\mathbf{X}(t ; \mathbf{x}, s), t)=f(\mathbf{X}(s ; \mathbf{x}, s), s)=f(\mathbf{x}, s)
$$

for any times $t$ and $s$ and phase space coordinate $\mathbf{x}$. It is this property which will be used in the semi-Lagrangian method to solve a discrete problem, which is defined by introducing a finite set of mesh points $\left(\mathbf{x}_{m}\right)_{m=1, . ., N}$ which may or may not be equally spaced. Then, given the value of the function $f$ at the mesh points at any given time step, we obtain the new value at mesh point $\mathbf{x}_{m}$ using that

$$
f\left(\mathbf{x}_{m}, t_{n}+\Delta t\right)=f\left(\mathbf{X}\left(t_{n}-\Delta t ; \mathbf{x}_{m}, t_{n}+\Delta t\right), t_{n}-\Delta t\right) .
$$

For each mesh point $\mathbf{x}_{m}, f$ is computed in two steps:

1. Find the starting point of the characteristic ending at $\mathbf{x}_{m}$, i.e. $\mathbf{X}\left(t_{n}-\Delta t ; \mathbf{x}_{m}, t_{n}+\Delta t\right)$

2. Compute $f\left(\mathbf{X}\left(t_{n}-\Delta t ; \mathbf{x}_{m}, t_{n}+\Delta t\right), t_{n}-\Delta t\right)$ by interpolation, $f$ being know only at mesh points at time $t_{n}-\Delta t$.

In the case of the 1D Vlasov-Poisson system (1)-(2), the numerical scheme described in the introduction actually involves these two steps. But, since the advection field for each half time step does not depend on the variable to be advected, step 1 is straightforward.

Now, for the general case, in order to deal with step 1, we need to introduce a time discretisation of (12). As in general no information on the advection function $U$ is known at any given time, we need to use a two time step scheme in order to remain second order in time. The starting point of the characteristic is obtained, to second order accuracy, by

$$
\frac{\mathbf{x}_{m}-\mathbf{X}\left(t_{n}-\Delta t\right)}{2 \Delta t}=\mathbf{U}\left(\mathbf{X}\left(t_{n}\right), t_{n}\right)
$$

writing, still to second order accuracy

$$
\mathbf{X}\left(t_{n}\right)=\frac{\mathbf{X}\left(t_{n}+\Delta t\right)+\mathbf{X}\left(t_{n}-\Delta t\right)}{2}
$$

there exists $\mathbf{d}_{m}$ such that $\mathbf{X}\left(t_{n}\right)=\mathbf{x}_{m}-\mathbf{d}_{m}$ and $\mathbf{X}\left(t_{n}-\Delta t\right)=\mathbf{x}_{m}-2 \mathbf{d}_{m}$. Then (13) becomes

$$
\mathbf{d}_{m}=\Delta t \mathbf{U}\left(\mathbf{x}_{m}-\mathbf{d}_{m}, t_{n}\right)
$$

which can be solved iteratively for the unknown $\mathbf{d}_{m}$.

Once $\mathbf{d}_{m}$ is known $f\left(\mathbf{x}_{m}-2 \mathbf{d}_{m}, t_{n}-\Delta t\right)$ is interpolated using a tensor product of cubic Bsplines.

Remark 4.1 Let us recall a few properties of the semi-Lagrangian methods that have been derived in previous investigations, see [6].

$\mathrm{RR} \mathrm{n}^{\circ} 3393$ 
- If $U$ is known independently of $f$ a one time step method can be used by introducing an intermediate time step.

- The time step is not restricted by the Courant condition. In practice accuracy conditions impose the time step which is usually of a few Courant time steps.

- Cubic interpolation appears to be a good compromise between accuracy and cost. Linear interpolation is too dissipative to be used.

- When using cubic interpolation for $f$, linear interpolation is sufficient for $U$.

- R. Bermejo [7] interpreted this method as a finite element PIC method and proved its convergence.

\section{Implementation of the guiding-centre model}

Let us now give a few more details on the implementation of the semi-Lagrangian method in the twodimensional case of the guiding-centre model which is a case where all the additional difficulties we are investigating are concentrated: The time-splitting cannot be performed so that we need a full two-dimensional scheme, the advection field, which is in this case the drift velocity, depends on $\mathbf{x}$. Moreover, passing to a higher dimension does not add any new difficulty. So the implementation of this specific case is a good benchmark for our method.

The model we consider is the following: the guiding-centre Vlasov equation

$$
\frac{\partial \rho}{\partial t}+\mathbf{v}_{D} \cdot \nabla_{x} \rho=0
$$

where

$$
\mathbf{v}_{D}=\frac{\mathbf{E} \times \mathbf{B}}{B^{2}}
$$

coupled to the Poisson equation

$$
-\Delta \phi=\rho
$$

with $\mathbf{E}=-\nabla \phi$, $\mathbf{B}$ being a given external magnetic field.

In this case the function $\mathbf{U}$ of the previous section is the drift velocity $\mathbf{v}_{D}=\mathbf{E} \times \mathbf{B} / B^{2}$ whose coordinates we denote by $v_{D x}$ and $v_{D y}$.

\subsection{Computation of the origin of the characteristics}

Applying the two-time step scheme as described in the previous section, the problem comes down to solving the fixed point equation (14). Denoting by $\left(\alpha_{i j}, \beta_{i j}\right)$ the coordinates of the displacement $\mathbf{d}_{m}$ at the mesh point $\mathbf{x}_{m}$ whose coordinates are denoted by $\left(x_{i}, y_{j}\right)$, equation (14) becomes

$$
\begin{aligned}
\alpha_{i j} & =\Delta t v_{D x}\left(x_{i}-\alpha_{i j}, y_{j}-\beta_{i j}, t_{n}\right) \\
\beta_{i j} & =\Delta t v_{D y}\left(x_{i}-\alpha_{i j}, y_{j}-\beta_{i j}, t_{n}\right)
\end{aligned}
$$

In order to solve this non linear system (17)-(18), as $\alpha_{i j}$ and $\beta_{i j}$ are small, the first obvious approach is to take

$$
v_{D x}\left(x_{i}-\alpha_{i j}, y_{j}-\beta_{i j}, t_{n}\right)=v_{D x}\left(x_{i}, y_{j}, t_{n}\right)
$$


and

$$
v_{D y}\left(x_{i}-\alpha_{i j}, y_{j}-\beta_{i j}, t_{n}\right)=v_{D y}\left(x_{i}, y_{j}, t_{n}\right) .
$$

However, this method being only first order accurate in $\Delta t$, it will not be convenient, or at least, as we shall see later in the numerical test, it would require much smaller time steps. In order to remain second order accurate, the system can be solved iteratively by the following procedure

$$
\begin{aligned}
& \alpha_{i j}^{k+1}=\Delta t v_{D x}\left(x_{i}-\alpha_{i j}^{k}, y_{j}-\beta_{i j}^{k}, t_{n}\right), \\
& \beta_{i j}^{k+1}=\Delta t v_{D y}\left(x_{i}-\alpha_{i j}^{k}, y_{j}-\beta_{i j}^{k}, t_{n}\right),
\end{aligned}
$$

starting from an initial guess $\left(\alpha_{i j}^{0}, \beta_{i j}^{0}\right)$.

This scheme can be proved to be convergent for $\Delta t$ small enough. Notice that $\mathbf{v}_{D}$, known only on the mesh, needs to be interpolated at the point $\left(x_{i}-\alpha_{i j}^{k}, y_{j}-\beta_{i j}^{k}\right)$. Numerical experiments performed for climate equations [6] have proved that linear interpolation is sufficient.

Using the Ansatz that $\mathbf{v}_{D}$ is linear in each grid cell, we also tried the more sophisticated Newton method in order to compute the solution of (17)-(18). The algorithm reads:

$$
\left(\begin{array}{c}
\alpha_{i j}^{k+1} \\
\beta_{i j}^{k+1}
\end{array}\right)=\left(\begin{array}{c}
\alpha_{i j}^{k} \\
\beta_{i j}^{k}
\end{array}\right)-\left(D f\left(\alpha_{i j}^{k}, \beta_{i j}^{k}\right)\right)^{-1} f\left(\alpha_{i j}^{k}, \beta_{i j}^{k}\right) .
$$

where

$$
f\left(\alpha_{i j}^{k}, \beta_{i j}^{k}\right)=\left(\begin{array}{c}
\alpha_{i j}^{k}-\Delta t v_{D_{x}}\left(x-\alpha_{i j}^{k}, y-\beta_{i j}^{k}\right) \\
\beta_{i j}^{k}-\Delta t v_{D y}\left(x-\alpha_{i j}^{k}, y-\beta_{i j}^{k}\right)
\end{array}\right),
$$

and the Jacobian matrix of $f$ is given by

$$
D f\left(\alpha_{i j}^{k}, \beta_{i j}^{k}\right)=\left(\begin{array}{cc}
\frac{\partial f_{x}}{\partial \alpha_{i j}^{k}} & \frac{\partial f_{x}}{\partial \beta_{i j}^{k}} \\
\frac{\partial f_{y}}{\partial \alpha_{i j}^{k}} & \frac{\partial f_{y}}{\partial \beta_{i j}^{k}}
\end{array}\right)=\left(\begin{array}{cc}
1+\Delta t \partial_{x} v_{D x} & \Delta t \partial_{y} v_{D x} \\
\Delta t \partial_{x} v_{D y} & 1+\Delta t \partial_{y} v_{D y}
\end{array}\right),
$$

where we omitted to indicate explicitly the dependence of $\mathbf{v}_{D}$ on $\alpha_{i j}^{k}$ and $\beta_{i j}^{k}$ for the sake of clarity. Then $\left(D f\left(\alpha_{i j}^{k}, \beta_{i j}^{k}\right)\right)^{-1} f\left(\alpha_{i j}^{k}, \beta_{i j}^{k}\right)$ can be computed using Cramer's formulae for a $2 \times 2$ system, which yields the algorithm

$$
\begin{aligned}
\alpha_{i j}^{k+1}= & \alpha_{i j}^{k}-\frac{1}{\Delta}\left[\left(\alpha_{i j}^{k}-\Delta t v_{D x}\left(x-\alpha_{i j}^{k}, y-\beta_{i j}^{k}\right)\right)\left(1+\Delta t \partial_{y} v_{D y}\left(x-\alpha_{i j}^{k}, y-\beta_{i j}^{k}\right)\right)\right. \\
& \left.-\left(\beta_{i j}^{k}-\Delta t v_{D y}\left(x-\alpha_{i j}^{k}, y-\beta_{i j}^{k}\right)\right) \Delta t \partial_{y} v_{D x}\left(x-\alpha_{i j}^{k}, y-\beta_{i j}^{k}\right)\right] \\
\beta_{i j}^{k+1}= & \beta_{i j}^{k}-\frac{1}{\Delta}\left[\left(\beta_{i j}^{k}-\Delta t v_{D y}\left(x-\alpha_{i j}^{k}, y-\beta_{i j}^{k}\right)\right)\left(1+\Delta t \partial_{x} v_{D x}\left(x-\alpha_{i j}^{k}, y-\beta_{i j}^{k}\right)\right)\right. \\
& \left.-\left(\alpha_{i j}^{k}-\Delta t v_{D x}\left(x-\alpha_{i j}^{k}, y-\beta_{i j}^{k}\right)\right) \Delta t \partial_{x} v_{D y}\left(x-\alpha_{i j}^{k}, y-\beta_{i j}^{k}\right)\right]
\end{aligned}
$$

where

$$
\begin{aligned}
\Delta= & \left(1+\Delta t \partial_{x} v_{D x}\left(x-\alpha_{i j}^{k}, y-\beta_{i j}^{k}\right)\right)\left(1+\Delta t \partial_{y} v_{D y}\left(x-\alpha_{i j}^{k}, y-\beta_{i j}^{k}\right)\right) \\
& -\Delta t^{2} \partial_{x} v_{D y}\left(x-\alpha_{i j}^{k}, y-\beta_{i j}^{k}\right) \partial_{y} v_{D x}\left(x-\alpha_{i j}^{k}, y-\beta_{i j}^{k}\right) .
\end{aligned}
$$

The values of $\mathbf{v}_{D}$ and its derivatives which are needed in this algorithm are computed using its mesh point values and the Ansatz that it is linear in each cell.

$\mathrm{RR} \mathrm{n}^{\circ} 3393$ 


\subsection{The two-dimensional spline interpolation}

Once $\alpha_{i j}$ and $\beta_{i j}$ are known $\rho\left(x_{i}-2 \alpha_{i j}, x_{j}-2 \beta_{i j}\right)$ is interpolated using a tensor product of cubic B-splines. In order to do this we first need to compute the coefficients $\eta_{\nu \kappa}$ of the cubic spline interpolation function $s(x, y)$ given by:

$$
s(x, y)=\sum_{-2 \leq \nu \leq N_{x}-1}\left(\sum_{-2 \leq \kappa \leq N_{y}-1} \eta_{\nu \kappa} B_{3 \nu}(x) B_{3 \kappa}(y)\right)
$$

The spline $s$ must satisfy the interpolation conditions

$$
s\left(x_{i}, y_{j}\right)=\rho\left(x_{i}, y_{j}, t_{n}-\Delta t\right)
$$

for $i=1, \ldots, N_{x}, j=1, \ldots, N_{y}$ and the two boundary conditions of the function $\rho$ in each direction, which in our case are periodic in the $x$-direction and natural in the $y$-direction.

In order to compute the $\eta_{\nu \kappa}$ coefficients, we first solve the $N_{y}$ one-dimensional interpolation problems:

$$
s\left(x, y_{j}\right)=\sum_{-2 \leq \nu \leq N_{x}-1} \gamma_{\nu}^{j} B_{3 \nu}(x) \text { for } j=1, \ldots, N_{y}
$$

each verifying the $N_{x}$ interpolation conditions $s\left(x_{i}, y_{j}\right)=\rho\left(x_{i}, y_{j}, t_{n}-\Delta t\right)$ and the periodic boundary conditions, where we denote by

$$
\gamma_{\nu}(y)=\sum_{-2 \leq \kappa \leq N_{y}-1} \eta_{\nu \kappa} B_{3 \kappa}(y)
$$

and by

$$
\gamma_{\nu}^{j}=\gamma_{\nu}\left(y_{j}\right)
$$

Using these interpolation and boundary conditions, we are brought to solve $N_{y}$ linear systems, one for each value of $j$, involving the same $\left(N_{x}+2\right)$-dimensional matrix which reads, denoting by $h=\Delta x$,

$$
\frac{1}{6}\left(\begin{array}{cccccccc}
-\frac{3}{h} & -\frac{3}{h} & 0 & \frac{3}{h} & \ldots & \ldots & \frac{3}{h} & 0 \\
-\frac{6}{h^{2}} & \frac{6}{h^{2}} & -\frac{12}{h^{2}} & \frac{6}{h^{2}} & \ldots & \ldots & -\frac{6}{h^{2}} & \frac{12}{h^{2}} \\
0 & 1 & 4 & 1 & & & & 0 \\
\vdots & 0 & 1 & 4 & \ddots & & & \vdots \\
\vdots & \vdots & & \ddots & \ddots & \ddots & & \vdots \\
\vdots & \vdots & & & \ddots & \ddots & \ddots & 0 \\
0 & \vdots & & & & 1 & 4 & 1 \\
1 & 0 & \ldots & \ldots & \ldots & 0 & 1 & 4
\end{array}\right) .
$$

Note that we have written this matrix down such that the first two rows correspond to the boundary conditions, the remaining $N_{x}$ to the interpolation conditions, and the $\left(N_{x}+2\right)$-dimensional unknown vector reads $\left(\gamma_{N_{x}-1}^{j}, \gamma_{-2}^{j}, \ldots, \gamma_{N_{x}-2}^{j}\right)$.

Then we obtain $\eta_{\nu \kappa}$ by solving the $N_{x}+2$ interpolation problems:

$$
\gamma_{\nu}(y)=\sum_{-2 \leq \kappa \leq N_{y}-1} \eta_{\nu \kappa} B_{3 \kappa}(y) \text { for } \nu=-2, \ldots, N_{x}-1,
$$


verifying the $N_{y}$ interpolation conditions $\gamma_{\nu}\left(y_{j}\right)=\gamma_{\nu}^{j}$ and natural boundary conditions. Using these interpolation and boundary conditions, we are brought to solve $N_{x}+2$ linear systems, one for each value of $\nu$, involving the same $\left(N_{y}+2\right)$-dimensional matrix which reads, denoting by $k=\Delta y$,

$$
\frac{1}{6}\left(\begin{array}{cccccccc}
-\frac{6}{k^{2}} & 0 & 0 & \cdots & \cdots & \cdots & \frac{6}{k^{2}} & -\frac{12}{k^{2}} \\
0 & \frac{6}{k^{2}} & -\frac{12}{k^{2}} & \frac{6}{k^{2}} & \cdots & \cdots & 0 & 0 \\
0 & 1 & 4 & 1 & & & & 0 \\
\vdots & 0 & 1 & 4 & \ddots & & & \vdots \\
\vdots & \vdots & & \ddots & \ddots & \ddots & & \vdots \\
\vdots & \vdots & & & \ddots & \ddots & \ddots & 0 \\
0 & \vdots & & & & 1 & 4 & 1 \\
1 & 0 & \cdots & \cdots & \cdots & 0 & 1 & 4
\end{array}\right) .
$$

This matrix is written for the $\left(N_{y}+2\right)$-dimensional unknown vector $\left(\eta_{\nu, N_{y}-1}, \eta_{\nu,-2}, \ldots, \eta_{\nu, N_{y}-2}\right)$ and the right-hand-side vector $\gamma_{\nu}^{N_{y}}, \gamma_{\nu}^{1}, \ldots, \gamma_{\nu}^{N_{y}-1}$.

In both matrices (19) and (20), the terms not explicitly written are all zeros except for fours on the diagonal and ones on the upper and lower diagonals.

Once the B-spline coefficients $\eta_{\nu \kappa}$ for all $\nu$ and $\kappa$ have been computed, the value of $\rho$ at the origin of the characteristics is taken to be the value of the B-spline $s\left(x_{i}-2 \alpha_{i j}, y_{j}-2 \beta_{i j}\right)$. If $\left(x_{i}-2 \alpha_{i j}, y_{j}-2 \beta_{i j}\right)$ belongs to $\left[x_{i}, x_{i+1}\right] \times\left[y_{j}, y_{j+1}\right]$ the approximation of the function $\rho\left(x_{i}-\right.$ $\left.2 \alpha_{i j}, y_{j}-2 \beta_{i j}, t_{n}-\Delta t\right)$ is given by:

$$
s\left(x_{i}-2 \alpha_{i j}, y_{j}-2 \beta_{i j}\right)=\sum_{i-3 \leq \nu \leq i}\left(\sum_{j-3 \leq \kappa \leq j} \eta_{\nu \kappa} B_{3 \nu}\left(x_{i}-2 \alpha_{i j}\right) B_{3 \kappa}\left(y_{j}-2 \beta_{i j}\right)\right)
$$

where:

$$
B_{3 \nu}(x)=\frac{1}{6 h^{3}} \begin{cases}\left(x-x_{\nu}\right)^{3} & x_{\nu} \leq x<x_{\nu+1} \\ h^{3}+3 h^{2}\left(x-x_{\nu+1}\right)+3 h\left(x-x_{\nu+1}\right)^{2}-3\left(x-x_{\nu+1}\right)^{3} & x_{\nu+1} \leq x<x_{\nu+2} \\ h^{3}+3 h^{2}\left(x_{\nu+3}-x\right)+3 h\left(x_{\nu+3}-x\right)^{2}-3\left(x_{\nu+3}-x\right)^{3} & x_{\nu+2} \leq x<x_{\nu+3} \\ \left(x_{\nu+4}-x\right)^{3} & x_{\nu+3} \leq x<x_{\nu+4}\end{cases}
$$

and $B_{3 \nu}(x)=0$ for $x<x_{\nu}$ and $x \geq x_{\nu+4}$. To compute $s\left(x_{i}-2 \alpha_{i j}, y_{j}-2 \beta_{i j}\right)$ for all $N_{x} N_{y}$ mesh points, requires $O\left(N_{x} N_{y}\right)$ floating point operations.

This algorithm can be fully parallelised. A description of the B-spline tensor product procedure of interpolation can be found in the books of De Boor [11], and H"ammerlin and Hoffmann [12].

\subsection{Numerical resolution of the 'almost' tridiagonal systems}

In order to compute the B-spline coefficients with the method described above, we are confronted with the resolution of linear systems which are tridiagonal except for two rows and columns. Instead of using full system solves, an ad hoc block decomposition of the matrices leads to a tridiagonal system solve coupled to a $2 \times 2$ system solve. All those matrices (19) and (20) have been brought by row and column exchanges to the following block form

$\mathrm{RR} \mathrm{n}^{\circ} 3393$

$$
M=\left(\begin{array}{cc|c}
\zeta_{1} & \zeta_{2} & \gamma^{T} \\
\zeta_{3} & \zeta_{4} & \\
\hline \gamma & A
\end{array}\right)
$$


where $\gamma$ is a matrix with two columns and $A$ a square positive definite tridiagonal matrix.

Then the block linear system

$$
M\left(\begin{array}{l}
x \\
y
\end{array}\right)=\left(\begin{array}{l}
b \\
c
\end{array}\right)
$$

can be solved using the following procedure: First, denoting by

$$
\delta=\left(\begin{array}{ll}
\zeta_{1} & \zeta_{2} \\
\zeta_{3} & \zeta_{4}
\end{array}\right)
$$

we can eliminate $y$, this gives the system

$$
\left(\delta-\gamma^{T} A^{-1} \gamma\right) x=b-\gamma^{T} A^{-1} c .
$$

Then $x$ being known, we compute $y$ by resolving

$$
A y=c-\gamma x .
$$

The system (22) is a $2 \times 2$ system which can be solved explicitly.

We are now ready to write down the numerical algorithm: Let us first notice that only the right hand side of the system (21) is time dependant. Hence we can factorise the matrix $A$ and assemble the $2 \times 2$ matrix (22) once for all in an initialisation step.

1. Initialisation

- factorise $A$ and store it

- compute and store $A^{-1} \gamma$ using the previously computed factorisation

- assemble the matrix $\delta-\gamma^{T} A^{-1} \gamma$

2. Time loop

- compute $A^{-1} c$ using the stored factorisation of $A$

- assemble $b-\gamma^{T} A^{-1} c$

- solve (22) using the explicit formulae

- compute $y$ using the already compute values of $A^{-1} c, A^{-1} \gamma$ and of $x$

The factorisation of $A$ and the subsequent resolutions of the systems involving $A$, which involves a large part of the computations performed in the code, are performed using optimised library subroutines.

\subsection{The time marching scheme}

After having detailed the implementation of the semi-Lagrangian method for the guiding-center Vlasov equations let us now write down the time marching algorithm for the full guiding-center Vlasov-Poisson problem:

Start with an initial charge density $\rho_{0}(x, y)$. In order to initialise our two time step advance, we first need to get the value of $\rho$ at the first time step. For this purpose, we perform a Poisson solve which yields $\mathbf{E}_{0}$, compute the drift velocity $\mathbf{v}_{D 0}$ and use equation (13) over half a time step using $\mathbf{v}_{D 0}$ as an approximation of $\mathbf{v}_{D}\left(t=\frac{1}{2} \Delta t\right)$ in order to get $\rho_{1}$.

Then $\rho_{n-1}$ and $\rho_{n}$ being given the time loop reads 
1. Compute $\mathbf{E}_{n}$ with a Poisson solve from $\rho_{n}$.

2. Compute the drift velocity $\mathbf{v}_{D n}$ from $\mathbf{E}_{n}$.

3. Compute $\rho_{n+1}$, using the two time step semi-Lagrangian algorithm, from $\rho_{n-1}$ and $\mathbf{E}_{n}$.

\section{The Kelvin-Helmholtz instability in a plasma}

In order to validate our code, we used two problems introduced by Ghizzo et al. [13].

\subsection{First test case}

First, we took a case where the growth rate of the instability can be computed analytically. This enabled us to check the accuracy of the code by comparing the analytical values with the computed ones.

Shoucri's analysis [14] shows how to start a Kelvin-Helmholtz instability for our model (15)(16) by picking an initial condition of the form $\rho(x, y, t=0)=\rho^{0}(y)+\epsilon \rho^{1}(y) \cos (k x)$ which yields through Poisson's equation a $\phi$ of the form $\phi=\phi^{0}(y)+\epsilon \phi^{1}(y) \cos (k x)$. The instability occurs by choosing properly the function $\rho^{1}$ which defines the perturbation around the equilibrium solution $\left(\rho^{0}, \phi^{0}\right)$. Shoucri picks $\rho^{0}(y)=\sin y$. Then he shows that taking $\phi^{1}=\psi_{s}=\sin \frac{y}{2}$, which he calls the neutrally stable solution, and which amounts to taking $\rho^{1}=\left(k^{2}-\frac{1}{4}\right) \sin \frac{y}{2}$, the perturbation should remain constant for all time $t$ if $k=k_{s}=\sqrt{3} / 2$, there should be exponential growth for $k<k_{s}$ and no instability for $k>k_{s}$. Moreover the growth rate of the instabilities should be such that

$$
\frac{\operatorname{Im}(\omega)}{k}=\frac{\sqrt{3}}{2}\left(k_{s}-k\right) .
$$

In order to compare our code with this theory, we ran two simulations with the initial condition

$$
\rho(x, y, t=0)=\sin y+0.015 \sin \frac{y}{2} \cos (k x),
$$

where $k=2 \pi / L_{x}, L_{x}$ being the length of the domain in the $x$-direction. In both runs the domain in the $y$-direction went from 0 to $2 \pi$, the number of mesh points in each direction was 128 and the time step 0.5 .

In the first case we took $L_{x}=7$ which yields $k=2 \pi / 7=0.89>k_{s}=\sqrt{3} / 2=0.86$. Hence we should be in the stable case. The results of this run are displayed in Figure 3, which clearly shows that there is no instability. In Figure 4 we also see that the first mode is not growing.

In the second case we took $L_{x}=10$ which yields $k=2 \pi / 10=0.62<k_{s}=\sqrt{3} / 2=0.86$. This time we should be in the unstable case, which is confirmed by the results displayed in Figure 1. Figure 4 also shows that the first mode is growing. Moreover the growth rate of the instability $\operatorname{Im}(\omega)$, according to equation (24) should have a value of 0.129 , for $k_{s}=\sqrt{3} / 2$ and $k=2 \pi / 10$. In Figure 5 where we zoom on the linear part of the temporal behaviour of the first mode, the slope for $\log \rho_{k}^{2}$ is 0.248 . This should be twice the value we are looking for. The computed growth rate is 0.124 , which is in very good agreement with the theoretical value.

In this case, we also displayed the evolution of the energy $\int E^{2} d x$ and enstrophy $\int \rho^{2} d x$, which are theoretically invariants of the system, in Figure 2. As expected for a semi-Lagrangian Vlasov code, see [3], the energy decreases during the smoothing phase, where the microstructures can not $\mathrm{RR} \mathrm{n}^{\circ} 3393$ 
be resolved within a cell and thus get smeared out. Except for this phenomenon, which is not unphysical although it does not appear in the Vlasov equation, the energy is very well conserved over many time-steps.

\subsection{Second test case}

In the second test case, still following Ghizzo et al. [13], we started the computation with an initial charge density

$$
\rho(x, y, t=0)=1.5 \operatorname{sech}(y / 0.9)\left(1+0.08 \sin \left(2 k_{0} x\right)\right)
$$

in order to start a Kelvin-Helmholtz instability. The computational mesh consisted of 128 points in each direction with $0 \leq x \leq 40$ and $-5 \leq y \leq 5$. We used a time step of 0.5 in the dimensionless units.

In order to solve the fixed point equation (14), we tried the three different procedures we described in Section 5.1:

1. Fixed point iterations which took around 6 iterations to converge.

2. The Newton method which took only 2 iterations to converge after the first time step. For both of these methods we kept the values of $\alpha$ and $\beta$ from the previous time step to start the iterations.

3. Doing no iterations at all assuming that $U\left(x_{i}-\alpha_{i j}, y_{j}-\beta_{i j}\right)$ was close enough to $U\left(x_{i}, y_{j}\right)$. This worked fine provided we divided the time step by 10 .

Through the choice of the initial condition, we excited the second mode. Hence the instability started right from the beginning of the run and saturated around time $t=20$ as we can see on Figure 9 yielding two circular rolling-up vortex structures. When the microstructures become of the order of the mesh size, smoothing occurs, see Figure 10. Then after some time a second instability takes place, Figure 11, where the two distinct structures merge and roll-up around each other. Here again, there are microstructures which smooth out when they reach the scale of the mesh size, as we can see on Figure 12. This second instability can be explained by the fact that the two vortex structure is an unstable equilibrium state. Mode 1 acts as a perturbation, and at some time depending on the discrete data the equilibrium is lost and the second instability takes place until the second mode vanishes as we can see on Figure 8.

As represented on Figures 6 and 7, the $L^{1}$ norm, i.e. $\int f d x d y$, even though it decreases slightly during the two unstable phases is conserved with an accuracy better than one percent. The $L^{2}$ norm, i.e. $\int f^{2} d x d y$, decreases strongly on two occasions and is stable the rest of the time, this is the same phenomenon as we discussed in the previous test case and which occurs when the microstructures get smoothed out as they become smaller than a cell size.

The first and second Fourier modes are also shown on Figure 8. The first mode shows a linear growth until the second instability and becomes steady afterward. The second mode growth during the first instability, remains steady until the second instability, and then decreases and stabilises at a smaller value. 


\section{Conclusion and perspectives}

In this paper, we introduced the semi-Lagrangian method for several types of Vlasov equations and discussed the simplifications that can be used in the implementation for some specific models where a time splitting procedure can be applied. On the other hand we described the full method which works for any type of Vlasov equation and implemented it for the case of the guiding-centre Vlasov-Poisson model which is an example which could not be solved accurately using the splitting procedure. The numerical results obtained on this example were very satisfying.

Building on the methods we introduced here we are now ready to develop a fortran 90 module library implementing the different kinds of advection types that are needed. Assembling these modules will then enable us to treat many problems occuring in plasma physics using the semiLagrangian methodology. Let us also mention that this methodology does not rely upon the use of regular grids. All we need is a set of lines in each direction for the spline interpolation, but these need not be equally spaced.

Acknowledgements The authors would like to acknowledge the Centre Charles Hermite in Nancy for computer time allocation on the Origin 2000 super computer. A.G. and P.B. would like to acknowledge G. Knorr for fruitful discussions on the topic.

\section{References}

[1] A. Ghizzo, P. Bertrand, M. Shoucri, T.W. Johnston, E. Filjakow, M.R. Feix J. Comput Phys. 90, $431(1990)$

[2] A. Ghizzo, P. Bertrand, M.L. Begue, T.W. Johnston, M. Shoucri, IEEE Transaction on Plasma Science 24, 370 (1996)

[3] M.R. Feix, P. Bertrand, A. Ghizzo, in Kinetic Equations, B. Perthame ed.

[4] O. Coulaud, E. Sonnendr ucker, E. Dillon, P. Bertrand, A. Ghizzo, INRIA report (to appear).

[5] Cheng, G. Knorr, J. Comput Phys. 22, 330 (1976)

[6] A. Staniforth and J. C`ot'e, Monthly Weather Review 119 (1991).

[7] R. Bermejo, Numer. Math. 60 (1991).

[8] G.I. Marchuk Methods of Numerical Mathematics, Springer-Verlag New-York (1982).

[9] E. Fijalkow, E. Jamin, M. Feix, P. Bertrand, A. Ghizzo, M. Shoucri, 14th conference on the numerical simulations of plasmas, PT2, Annapolis (1991).

[10] M. Shoucri, IEEE Transaction on Plasma Science PS-7, (2) (1979).

[11] C. De Boor, A Practical Guide to Splines, Springer-Verlag New-York (1978).

[12] G. H“ammerlin, K.H. Hoffmann, Numerical Mathematics, Springer-Verlag New-York (1991).

[13] A. Ghizzo, P. Bertrand, M. Shoucri, E. Fijalkow, M.R. Feix J. Comput Phys. 108, 105 (1993)

[14] M. Shoucri, Internat.J. Numer. Meth. Eng. 17, 1525-1538 (1981).

$\mathrm{RR} \mathrm{n}^{\circ} 3393$ 

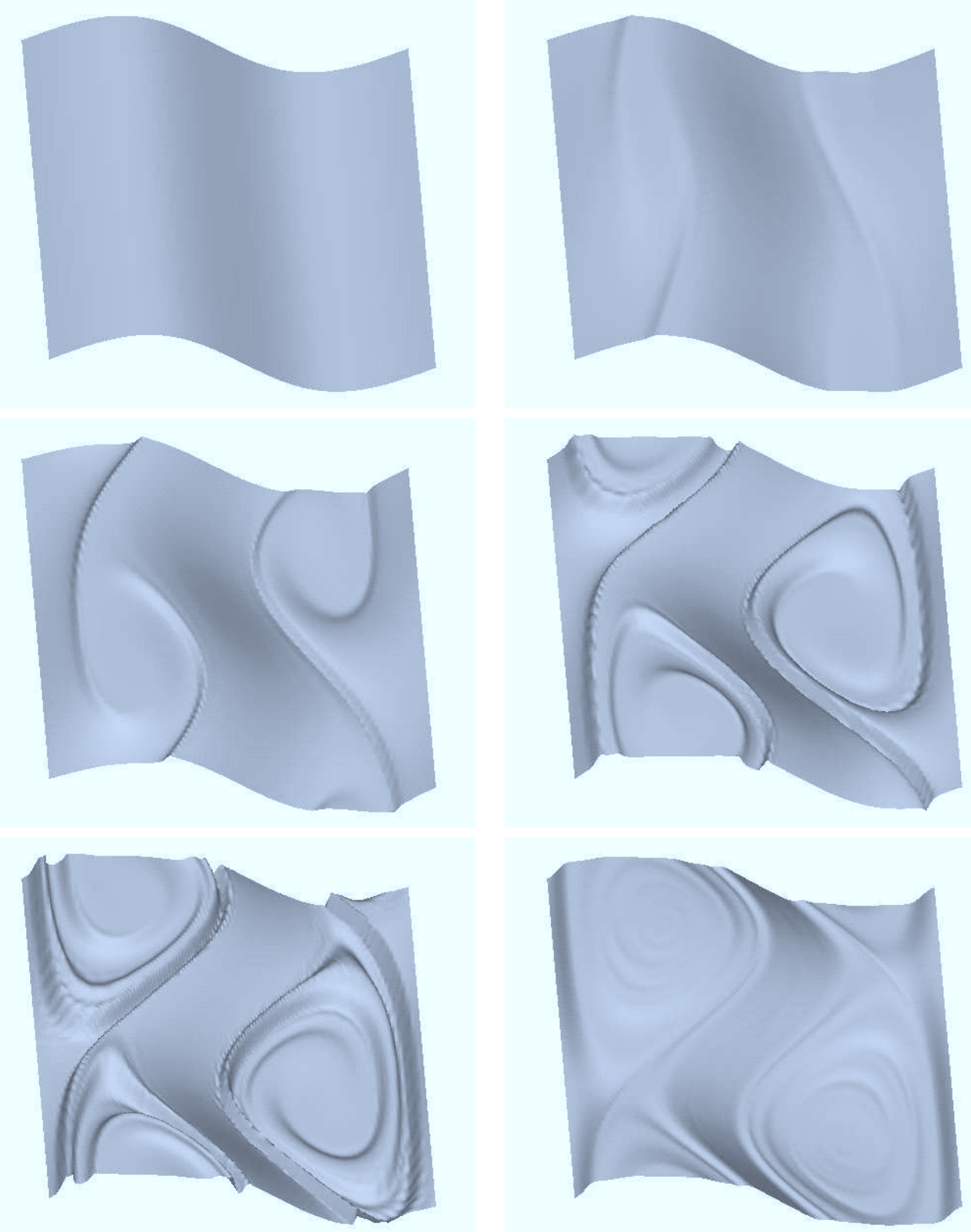

Figure 1: The unstable case: from left to right and top to bottom $t=0, t=20, t=30, t=40, t=50$ and $\mathrm{t}=1000$. 

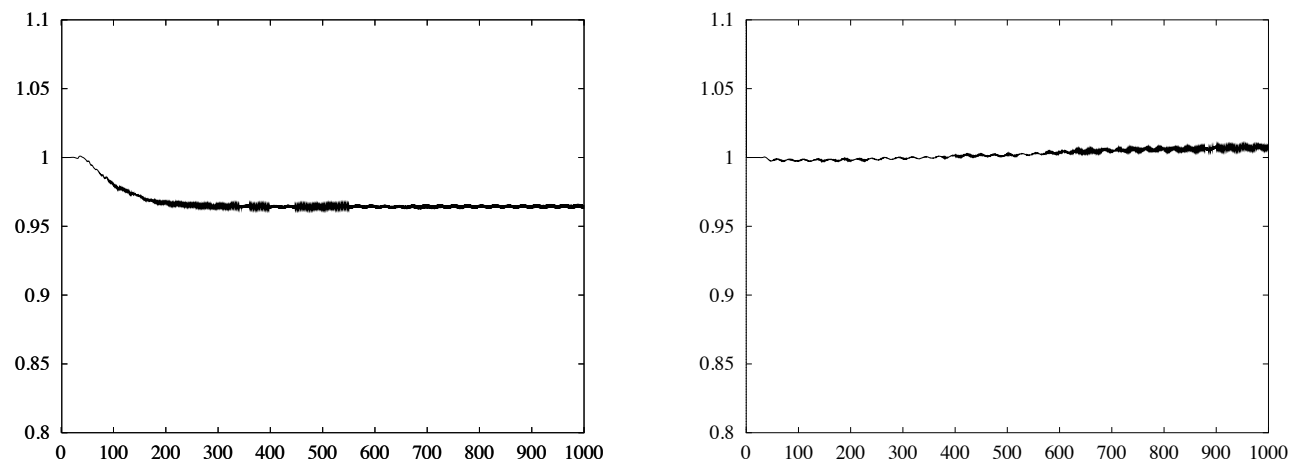

Figure 2: The invariants: energy (left) and enstrophy (right).
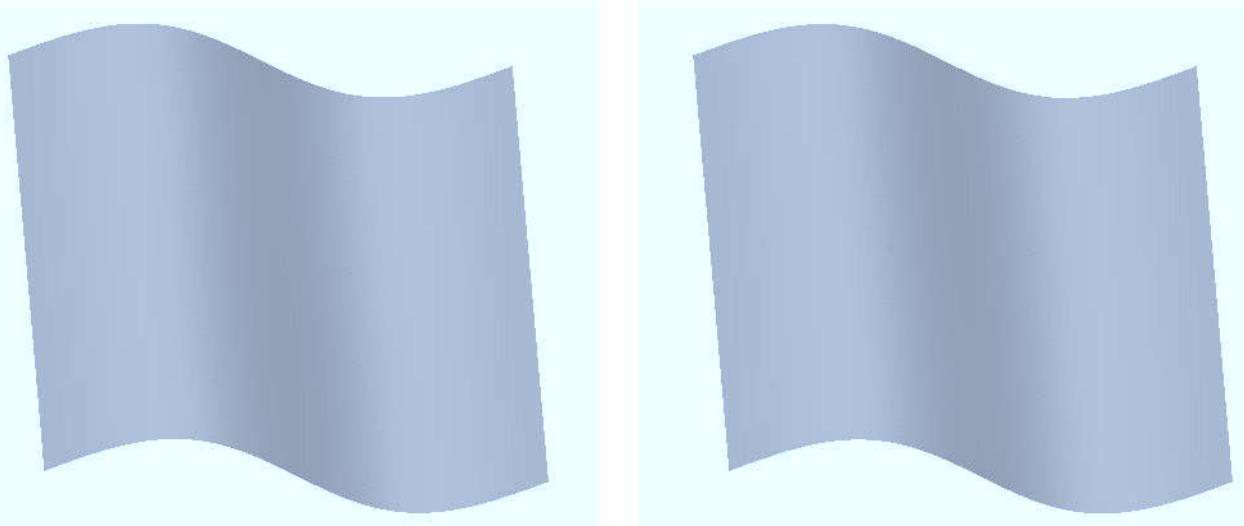

Figure 3: The stable case: $\mathrm{t}=0$ (left) and $\mathrm{t}=1000$ (right).
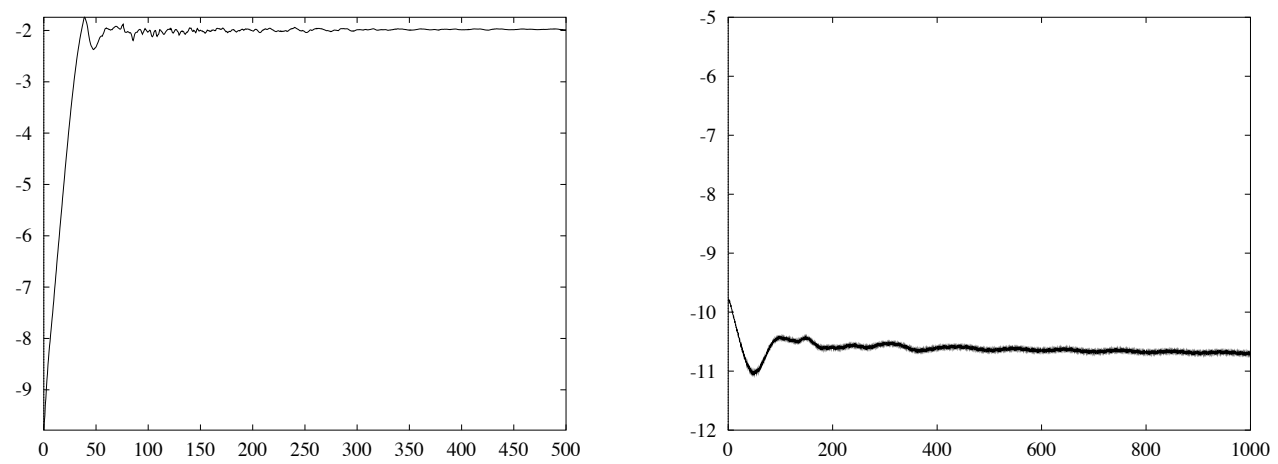

Figure 4: The first Fourier mode: $\log \rho_{k}^{2}$ with respect to time, unstable case (left) and stable case (right).

RR n` 3393 


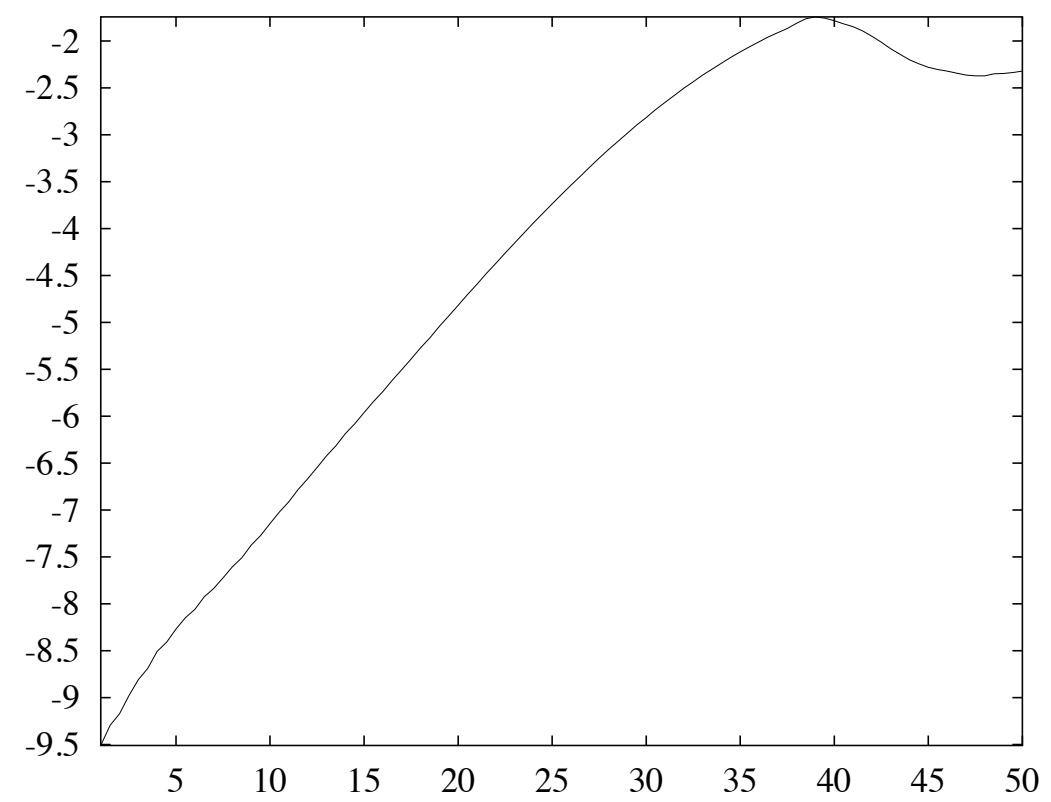

Figure 5: Computation of the growth rate of the linear part of the First Fourier mode.
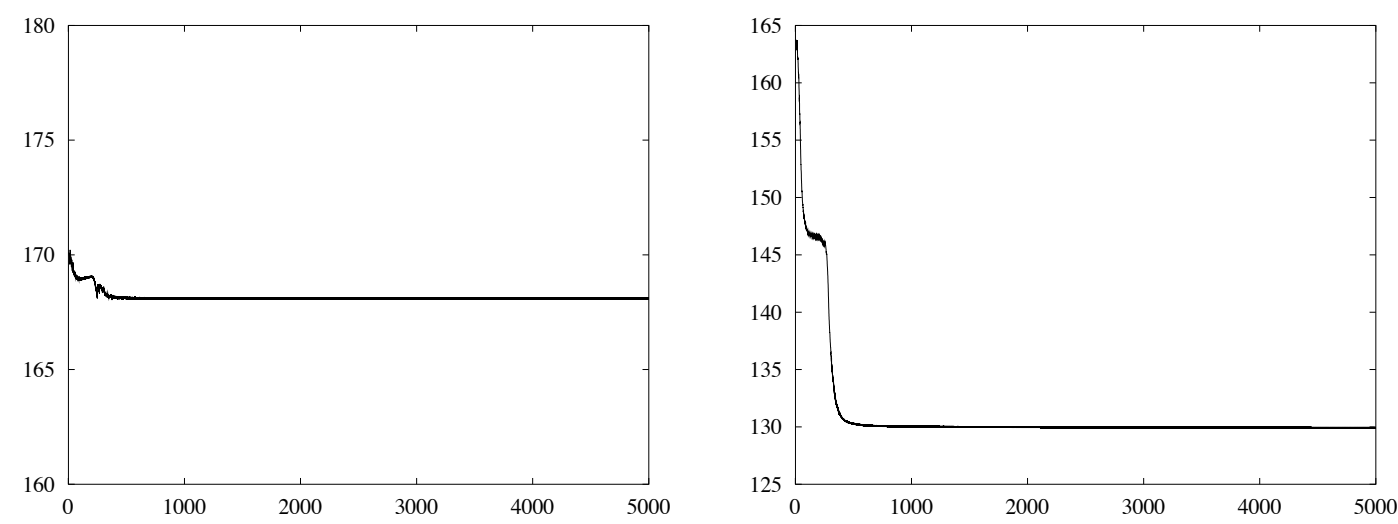

Figure 6: Graphs of the $L^{1}$ norm (left) and $L^{2}$ norm (right) with respect to time. 

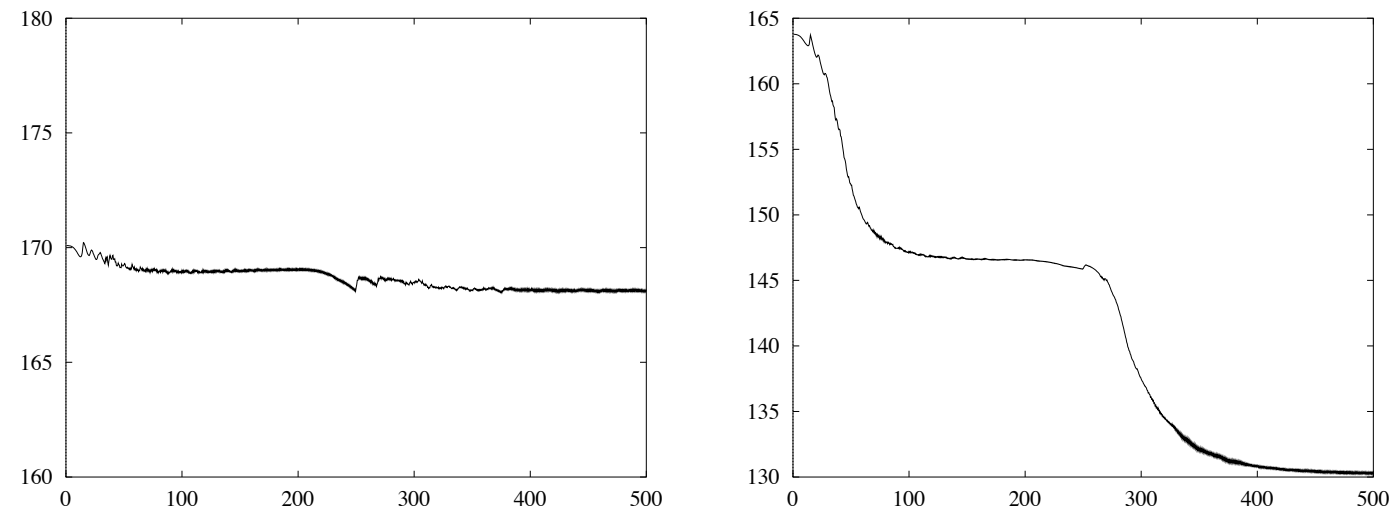

Figure 7: Evolution of the $L^{1}$ norm (left) and $L^{2}$ norm (right) with respect to time between $\mathrm{t}=0$ and $\mathrm{t}=500$ for the Kelvin-Helmholtz instability.
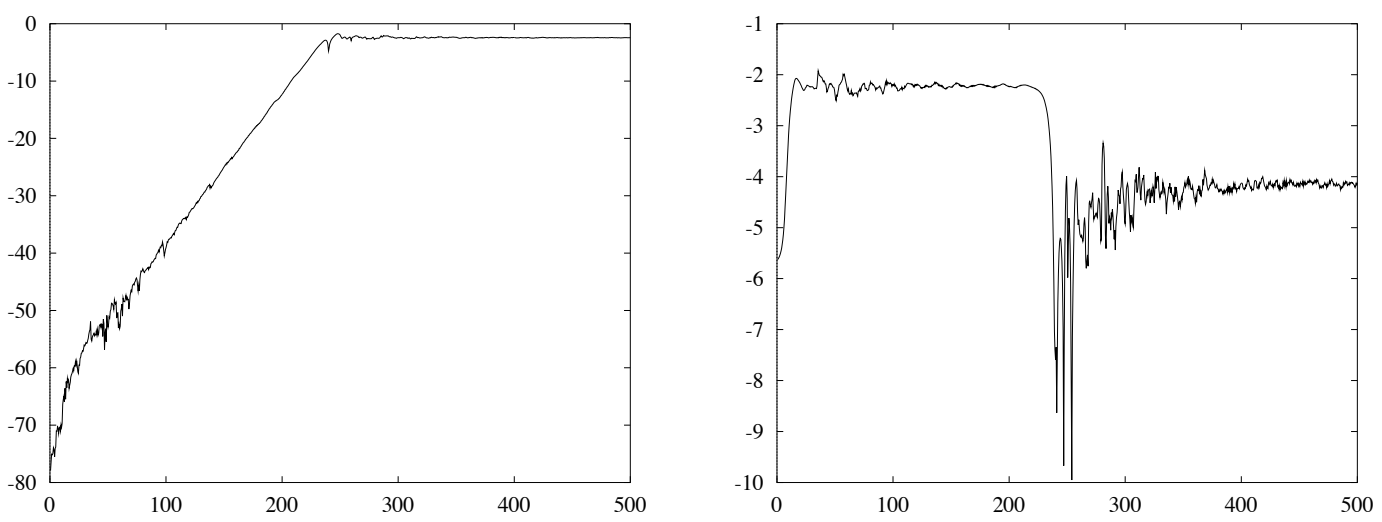

Figure 8: Evolution of $\log$ of the first (left) and second (right) Fourier modes with respect to time between $\mathrm{t}=0$ and $\mathrm{t}=500$.

$\operatorname{RR} n^{\circ} 3393$ 

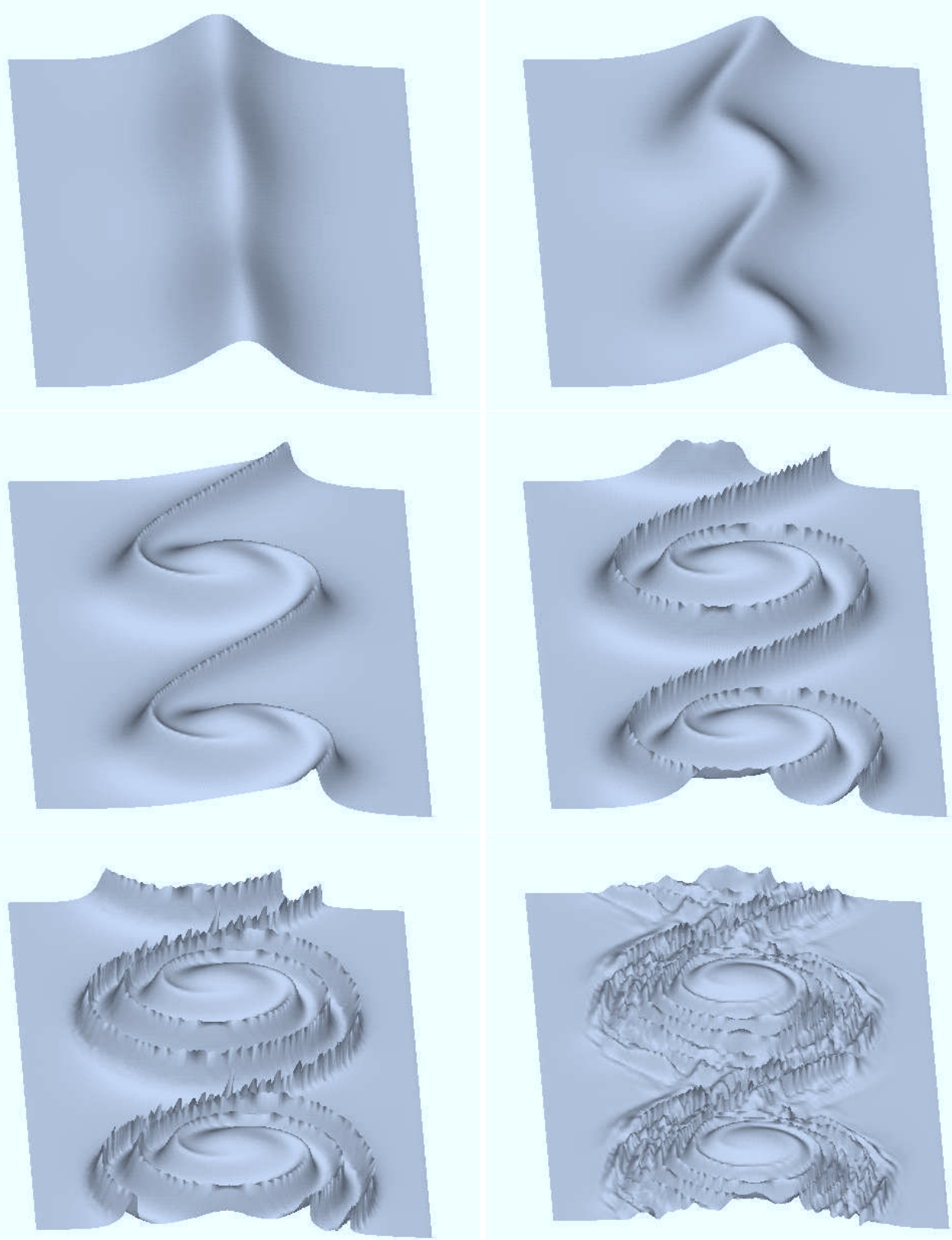

Figure 9: Development of the first instability, from left to right and top to bottom $t=0, t=5, t=10$, $\mathrm{t}=15, \mathrm{t}=20$ and $\mathrm{t}=50$ 

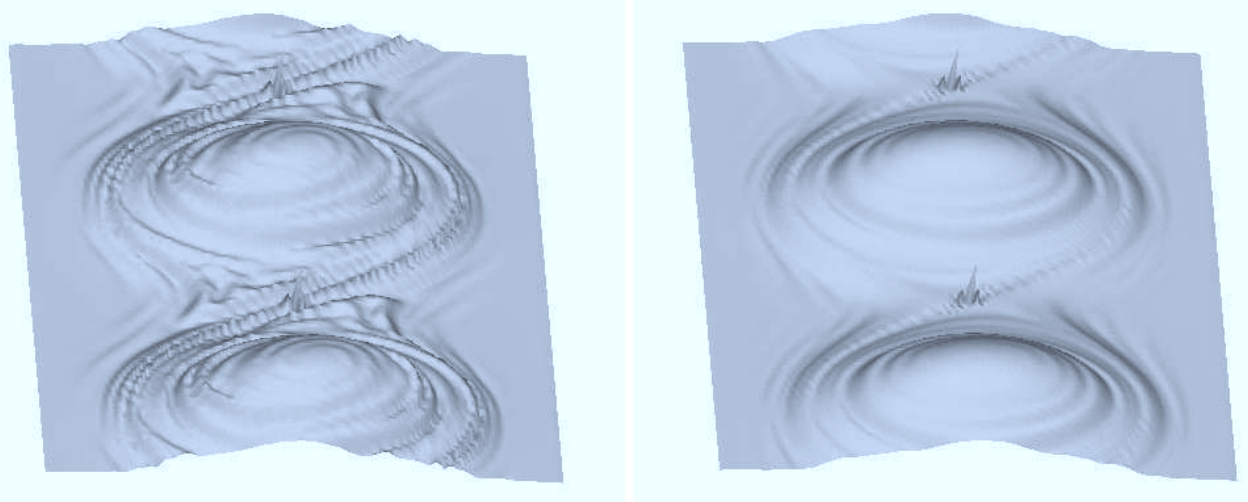

Figure 10: Between the instabilities, left $\mathrm{t}=100$ and right $\mathrm{t}=200$

RR n`3393 

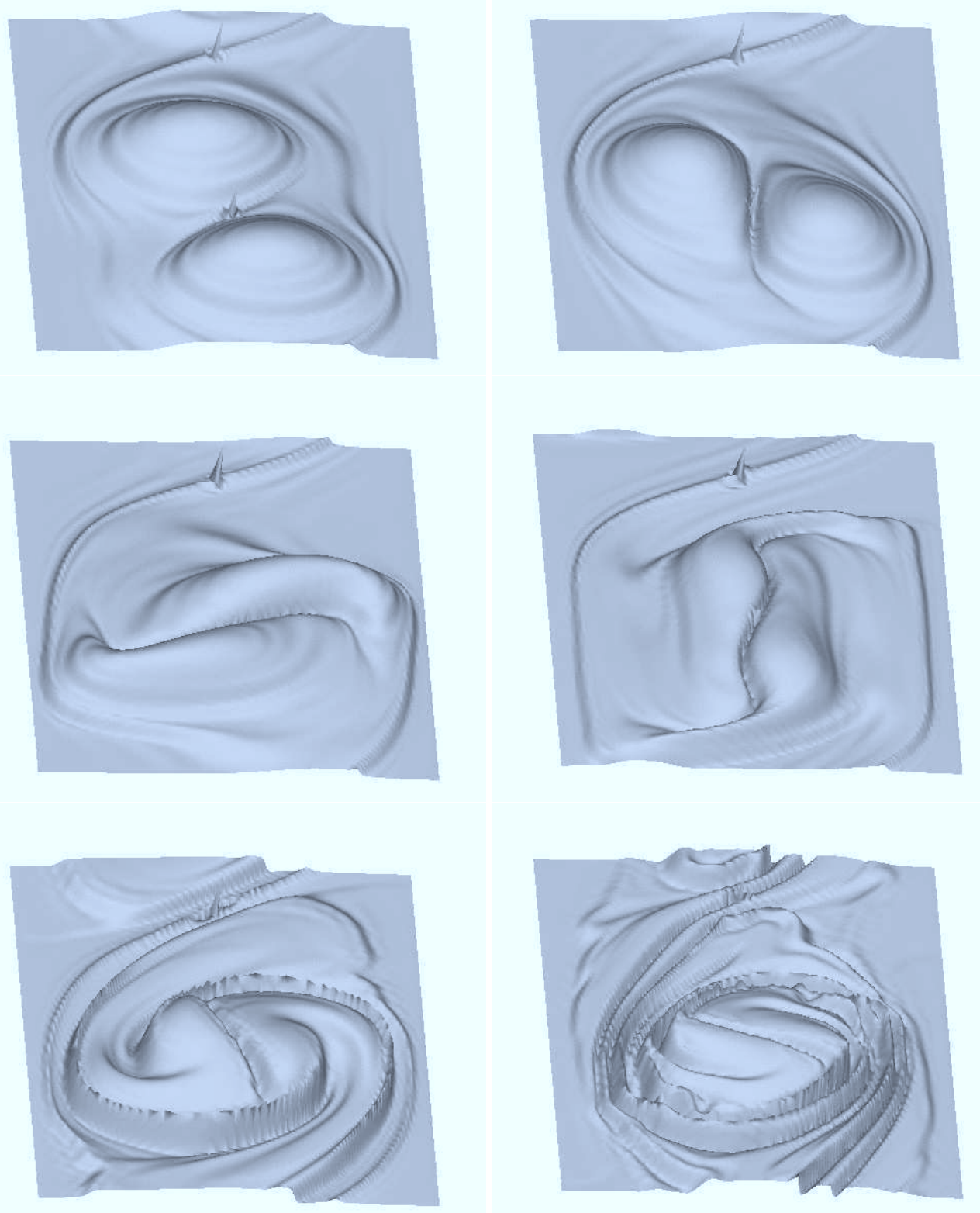

Figure 11: Development of the second instability, from left to right and top to bottom $t=235, t=240$, $\mathrm{t}=245, \mathrm{t}=250, \mathrm{t}=260$ and $\mathrm{t}=275$ 

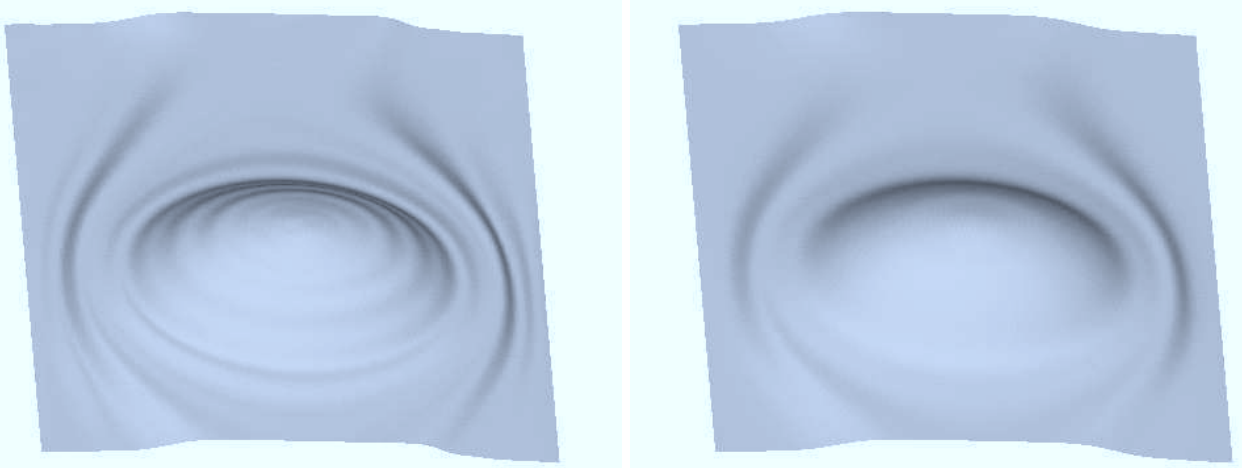

Figure 12: Steady-state, left $t=1000$ and right $t=5000$

$\operatorname{RR} n^{\circ} 3393$ 